\title{
Short term wind power forecasting using hybrid intelligent systems
}

\author{
M. Negnevitsky, Member, IEEE, P. Johnson, Student Member, IEE and S. Santoso, Senior Member, IEEE
}

\begin{abstract}
This panel paper summarizes the current trends in wind power development and describes a proposed approach for short term wind power forecasting using a hybrid intelligent system.
\end{abstract}

Index Terms-wind power, short term forecasting, intelligent systems.

\section{INTRODUCTION}

The publication of the Stern Review on the economics of climate change [1] on 30 October 2006 re-energized the public and political debate regarding the need for low carbon technologies for electricity power generation. The Review represented the most comprehensive study ever carried out on the economics of climate change and estimated that the global cost of a "business as usual" approach would be equivalent to at least 5\% of Gross Domestic Product (GDP) and could be as high as $20 \%$ of GDP each year. In contrast, the costs of action to reduce greenhouse gas emissions to avoid the worst impacts of climate change can be limited to around 1\% of GDP and the global benefits over time could be in the order of $\$ 2.5$ trillion each year.

Power generation accounted for $24 \%$ of greenhouse gas $\left(\mathrm{CO}_{2}\right)$ emissions in 2000. The Stern Review highlights that for electricity generation, where new technologies can struggle to gain a foothold, support policies for early stage, low carbon technologies will be critical.

Wind power is now a maturing renewable energy technology for electricity generation with various high efficiency, multi-megawatt turbine configurations commercially available to use as building blocks for utility scale wind farm installations.

Global installed wind power capacity continues to grow at around 25\% per year. This growth is expected to increase with continued political efforts and support policies for renewable energy. Figures compiled by the Global Wind Energy Council indicate that at the end of 2005 the total installed wind power capacity was 59,084MW worldwide [2].

While the European Union still leads the market with over 40,500MW installed at the end of 2005 (69\% of the global total), nearly a quarter of the new capacity installed in 2005 was in North America with the United States leading the way with over 2,400MW installed for the year. This growth in the US was due largely to the current window of stability in the federal incentive for wind energy, the production tax credit

M. Negnevitsky and P.L. Johnson are with the University of Tasmania, Hobart, Australia.

S. Santoso is with the Department of Electrical and Computer Engineering, The University of Texas at Austin, Austin, TX 78712, USA
(PTC). Other emerging markets include India with 1,430MW installed in 2005 and China with 496MW installed in 2005 in anticipation of that country's new Renewable Energy Law which came into force on 1 January 2006.

These current trends confirm that wind power is a significant and growing sector of renewable power generation and one which will continue to rise in profile, particularly as the global political will to address the worst impacts of climate change gains momentum.

With the growth in the popularity of wind power as a commercially viable, renewable energy resource, a number of problems arise with integration of that resource into existing power systems and electricity markets. This paper summarizes some of these problems and highlights the need for accurate short term forecasting of wind power generation. An overview of wind power forecasting techniques is provided and some issues related to prediction parameter choices are discussed. Finally the application of hybrid intelligent systems to short term wind power forecasting is discussed and a proposed research direction is outlined.

\section{WIND POWER AND ELECTRICITY MARKETS}

\section{A. The Nature of Wind Power}

Wind and the resulting power produced by wind turbines are neither constant nor schedulable. One of the fundamental difficulties faced by power system operators is the unpredictability and variability of wind farm generation. This has both technical and commercial implications for the efficient planning and operation of power systems[3].

The increasing penetration of wind generation into power systems gives rise to the need for greater forecasting predictability to maintain system security and enable assets to be operated in the most economical manner. Consequently the need exists for wind power prediction tools which offer the best possible accuracy over a number of time scales.

Wind farms are often located in remote areas which are serviced by "stringy" and constrained transmission networks. In such cases, the need for accurate forecasting of wind farm outputs is required to ensure appropriate loading and secure operation of the constrained transmission networks servicing them.

Data time series representing wind parameters and wind power are chaotic or stochastic in nature and this presents challenges for accurate prediction, particularly in the shortterm time frame. However, accurate wind power forecasting is necessary to enable successful integration of wind generation into power systems and electricity markets. 


\section{B. Electricity Markets and Wind Power}

Many electricity markets around the world have moved from a regulated market supplied via vertically integrated power generation, transmission and distribution monopoly models to de-regulated (or 're-regulated') models with disaggregation of generators and distributors.

Deregulated electricity markets generally operate using either a bilateral contract arrangement and a nett wholesale electricity pool such as the New Electricity Trading Agreement (NETA) in the United Kingdom or using a gross wholesale electricity pool arrangement such as is employed in the Australian National Electricity Market (NEM). In each type of system there are mechanisms for energy sales on different time scales and a system to control dispatching to meet the technical system requirements.

The acceptance of non-schedulable power such as wind power into electricity markets is also handled in a variety of ways. In some cases, government regulated feed-in pricing is set for variable, renewable sources. In other markets, all participants must compete on equal terms.

In Australia, the wholesale electricity market operator responsible for system security and operation of the NEM is the National Electricity Market Management Company (NEMMCO). A large proportion of power is hedged via long term financial contracts between generators and retailers. NEMMCO sets the wholesale pool price for each five minute dispatch interval based on generator bidding and settlement takes place on a thirty minute trading interval basis.

Wind power is currently non-scheduled and accepted straight into the NEM with wind power generators able to receive the market pool price. In addition, wind farm developers can currently sell Renewable Energy Certificates (RECs) which retailers are required to buy under Australia's Mandatory Renewable Energy Target (MRET) scheme. The value of RECs also varies based on a supply and demand market.

For the purposes of ensuring system security and dispatching generation capacity to match demand, NEMMCO treats wind power as a 'negative demand'. This stochastic 'negative demand' subtracts from the actual peak load profile resulting in a modified peak demand. This approach links demand forecasting to wind power forecasting and consequently the importance of accurate short term wind power forecasting becomes more significant as the penetration of wind power increases.

In August 2006, NEMMCO published a set of functional requirements for the development of an Australian Wind Energy Forecasting System (AWEFS) [4]. These functional requirements highlight the importance of accurate short term wind power forecasting for the 5 minute dispatch interval and also call up short term forecast requirements for 11 subsequent 5 minute pre dispatch intervals out to 60 minutes ahead.

\section{WIND POWER FORECASTING}

\section{A. Overview of Forecasting Techniques and Time Scales}

Wind and wind power forecasting techniques available include numeric weather prediction (NWP) methods, statistical methods and methods based on the use of Artificial Neural Networks (ANNs).

The time frames used for traditional wind forecasting range from the planetary scale for extremely large weather patterns over the long term (months to years), to synoptic predictions for large weather patterns over the medium term (days to weeks) down to "mesoscale" forecasting for smaller areas with the following sub time scales defined:

mesoscale alpha (intermediate term: 6 hours -2 days)

mesoscale beta (short term: 30minutes -6 hours)

mesoscale gamma (very short term - less than 30 minutes)

NWP methods are well established for wind forecasting with a prediction horizon of several hours or more. Specifically tuned NWP models using accurate Digital Elevation Models (DEMs) and Model Output Statistics (MOS) corrections have been applied to short term wind forecasting applications with some success but they have proven to be unsuitable for the very short term time frame.

The available wind and wind power prediction techniques for the very short term (or mesoscale gamma) time frame rely on current local observations as model inputs. These techniques range from the "industry benchmark" persistence approach to statistical models and the application of Artificial Neural Networks.

The concept of persistence was developed by meteorologists as a simple short term forecasting approximation to augment NWP methods. Persistence relies on the high correlation between the current value of a wind parameter and the value in the immediate future and simply equates the next forecast value to the current value. This approach becomes less valid as the forecast interval increases but persistence has proven to be a useful first approximation for short term forecasting and provides a benchmark against which to gauge the accuracy of alternative techniques.

Some work has been undertaken on the application of neural networks to model and estimate wind turbine output [5, 6]. However the specific tuning required and lack of general portability of these approaches can be restrictive. To date no single approach has gained widespread industry acceptance and the simple persistence model is often used even though more accurate predictions can be achieved.

\section{B. Forecast Parameter Choices}

When considering short term wind power forecasting there are a number of choices in relation to which parameters to forecast and what level of aggregation to consider. The first choice relates to whether to forecast wind parameters and infer or calculate wind power from those predictions or alternatively to forecast wind power directly using a suitable model.

Forecasting wind parameters (e.g. wind speed or wind vector magnitude) is a more fundamental approach and the intermediate results of predicted wind parameter values will be independent of turbine type and performance. However, where the goal is to forecast wind power output, conversion of forecast wind parameters into power output is required.

The general relationship between wind speed and the output power of a wind turbine is given by: 
$P=\frac{1}{2} \cdot C_{p} \cdot \rho \cdot A \cdot v^{3}$

where $P$ is the output power $(W) ; C_{p}$ is the rotor coefficient of performance; $\rho$ is the air mass density $\left(\mathrm{kg} / \mathrm{m}^{3}\right) ; A$ is the swept blade area $\left(\mathrm{m}^{2}\right) ; v$ is the speed of the wind perpendicular to the turbine(m/s).

For a particular turbine, the coefficient of performance $\left(C_{p}\right)$ varies non linearly with wind speed and the overall turbine output performance is usually represented using a rating curve of power output vs. wind speed provided by the manufacturer. However a single rating curve does not adequately represent the dynamic response of a turbine output to varying wind speed and direction in the short term time frame. Consequently if wind parameters for a turbine are accurately forecast using a particular model, there is no simple relationship to obtain accurate power output from these parameters and a second non linear model will be required. This suggests that where short term wind power forecasting is required, a single model providing direct prediction of power output is the best approach.

Another forecasting parameter choice relates to the level of aggregation to be considered. The most flexible approach is to consider short term wind power forecasting on a per turbine basis. By producing a forecasting model for each turbine, the output for a wind farm can be easily scaled and adjusted depending on turbine availability and wind farm configuration changes over time. In addition, inputs which are local and specific to each turbine such as wind speed at the hub and current turbine orientation in relation to the wind direction (yaw angle) could be considered in the model.

Alternatively, aggregated short term wind power forecasting for a group of wind turbines may be easier to manage and may provide some inherent error cancellation. Aggregation could be considered for a sub-circuit of a wind farm or for an entire wind farm. While aggregation of a number of wind farms in a region could also be considered, this is expected to be less useful for short term forecasting where local effects will dominate for the time frames being considered.

\section{PROposed ApplicAtion OF Hybrid InTELligent SYSTEMS}

\section{A. Intelligent Systems Overview}

The field of Artificial Intelligence has found many applications in the operation of power systems. These applications range from Expert Systems to assist with network fault diagnosis and rectification to Artificial Neural Networks and Fuzzy Logic to provide models for complex non-linear control problems. Combinations of different intelligent system approaches to form hybrid intelligent systems continue to find new applications.

\section{B. Proposed Approach for Short Term Wind Power Forecasting}

Recent research has demonstrated the use of a novel technique to forecast wind vectors 2.5 minutes ahead [7]. An extension of this technique to predict single turbine power output and aggregated wind power output has also been successfully demonstrated over this time frame [8].

This innovative approach applies the combination of two Artificial Intelligence techniques, Fuzzy Logic and Artificial Neural Networks in the form of a hybrid model called an Adaptive Neural Fuzzy Inference System (ANFIS) to predict wind parameter and wind power output in the very short term time frame (currently out to 2.5 minutes ahead).

Building on this work, investigations are in progress to determine which model configurations provide the best accuracy for the forecasting of wind power over a 5 to 15 minute time frame. The intent is to develop a portable intelligent system capable of being employed for any wind farm (without the need for expert input) which will provide improved forecasting accuracy over this time frame. Following this, intelligent system approaches to wind power forecasting out to 30 minutes will be investigated. It is intended that the approaches developed will be tested using a number of wind farm case studies with a variety of wind characteristics and turbine configurations to confirm the portability of the models developed.

One of the wind farms is in North America. Fig. 1 below shows actual wind speed and wind power output measured at a collector bus. The data length shown is for a consecutive three day period. The wind speed data was measured at a single location within the wind farm and has a native resolution of 30 seconds. The wind power data has a much higher resolution, i.e., 1 second.
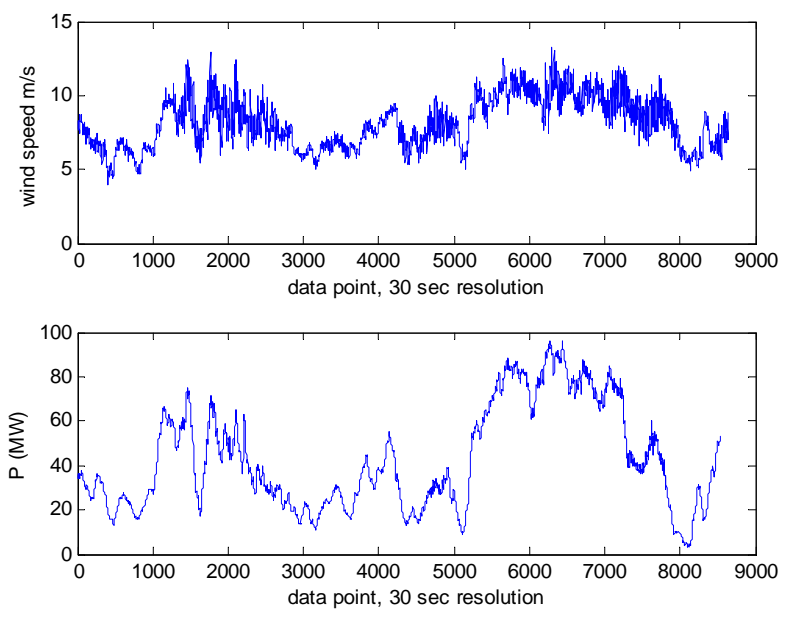

Fig. 1. Wind speed (top) and wind power (bottom) time-series. Note that valleys and hills in the time-series vary in nearly unison.

The plotted wind power data in Fig. 1 has been smoothed to 30 second resolution using a moving window approach to match the resolution of the wind speed. The total data length is 8640 data points (3 days). For this particular wind speed and power pair, wind power variation remarkably matches that of wind speed. One can easily notice that valleys and hills in the time-series correlate rather tightly. Since many wind farms can extend over several kilometers wide, wind speed over the wind farm area is not uniform. It is conceivable to have a strong gust at one end of the farm, with 
no or very little wind at the other end. The proposed forecasting tool will develop algorithms to deal with this phenomenon.

\section{REFERENCES}

[1] N. Stern. Stern Review: The Economics of Climate Change. 2006 [cited 10 November 2006]; Available from:

http://www.hmtreasury.gov.uk/independent_reviews/stern_review_econ omics_climate_change/stern_review_report.cfm.

[2] GWEC. Global Wind 2005 Report. [cited 10 November 2006]; Available from: http://www.gwec.net.

[3] G. N. Bathurst, J. Weatherill, and G. Strbac, "Trading wind generation in short term energy markets," IEEE Transactions on Power Systems, vol. 17, pp. 782-789, 2002.

[4] Functional requirements for AWEFS and NEM forecasting processes. National Electricity Market Management Company Limited.18 August 2006 [cited October 10 2006]; Version 1.0:[ROI support documentation]. Available from: http://www.nemmco.com.au/dispatchandpricing/awefs.ht m.

[5] S. Kelouwani and K. Agbossou, "Nonlinear model identification of wind turbine with a neural network," IEEE Transactions on Energy Conversion, vol. 19, pp. 607-612, 2004.

[6] L. Shuhui, D. C. Wunsch, E. A. O'Hair, and M. G. Giesselmann, "Using neural networks to estimate wind turbine power generation," IEEE Transactions on Energy Conversion, vol. 16, pp. 276-282, 2001.

[7] C. W. Potter and M. Negnevitsky, "Very short-term wind forecasting for Tasmanian power generation," IEEE Transactions on Power Systems, vol. 21, pp. 965-972, 2006.

[8] M. Negnevitsky, and Potter, C., "Innovative Short-Term Wind Generation Prediction Techniques”, Proceedings of the IEEE/PES General Meeting, Montreal, Canada, 18-22 June 2006, CD-ROM, IEEE Catalog Number 06CH37818C, ISBN 1-4244-0493-2.

Michael Negnevitsky (M’95) received his B.S.E.E. (Hons), 1978, and Ph.D., 1983, from Byelorussian University of Technology, Belarus.

Currently, he is a Professor in the School of Engineering at the University of Tasmania, Australia. From 1984 to 1991, he was a Senior Research Fellow and Senior Lecturer in the Department of Electrical Engineering, Byelorussian University of Technology, Belarus. After his arrival in Australia, Dr. Negnevitsky was with Monash University, Australia. His major interests are power system analysis, power quality, and intelligent systems applications in power systems. Dr. Negnevitsky is a Chartered Professional Engineer, a Senior Member of the Institution of Engineers Australia, and a Member of CIGRE AP C4 (System Technical Performance), Australian Technical Committee and a Member of CIGRE Australian Wind Energy Task Force.

Peter L. Johnson (StudM’06) received his B.E. (Hons) 1986, and M.Tech 1997, from the University of Tasmania, Australia. The author is currently a Ph.D. candidate with research interests in wind power, intelligent systems and power system operations.
He has worked as a power electronics design engineer with Critec Pty. Ltd, a research and development engineer with Global Lightning Technologies Pty. Ltd. and as senior electrical engineer with Pitt \& Sherry consulting engineers. Mr. Johnson is a Chartered Professional Engineer, a Member of the Institution of Engineers, Australia and a member of Standards Australia Committee EL/24 for Lightning Protection.

Surya Santoso (M'1996, SM 2002) earned his MSE and Ph.D. degrees in electrical and computer engineering from the University of Texas at Austin in 1994 and 1996, respectively. He re-joined the University of Texas at Austin as an assistant professor in fall 2003 after a seven-year stint as a senior and consulting engineer with Electrotek Concepts, Inc in Knoxville, TN. His primary research interests include development of intelligent systems for analyzing raw power quality measurement data, power system modeling and studies, and wind power. He co-authored "Electric Power Systems Quality", published by McGraw-Hill Professional Series in 2002. 